\title{
Nasal Endoscopic Appearance in Adult Patients with Idiopathic Epistaxis
}

Widanage W. H. U. P, Fernando R.

\section{Introduction}

Epistaxis is perhaps the most common ENT emergency ${ }^{(6)}$. A variety of aetiologies can cause epistaxis, including systemic and local causes. But most of the time there is no identifiable cause and labelled as idiopathic. This study aims to identify the incidence of usually known conditions such as bleeding from anterior inferior end of septum (little's area), Telangiectasia, AVM etc., and also to note other possible abnormal anatomy predisposing nasal bleeding in a Srilankan population.

The local treatment for the bleeding point is considered the most efficient and simplest way to manage epistaxis ${ }^{(2,3,4)}$. This will avoid all the complications associate with nasal packing and other invasive procedures. This is quite straightforward in 'anterior 'bleeding due to a bleeding point located at Little's area but it could be very challenging if it were in posterior nose. Commonest site of bleeding in posterior epistaxis is still controversial as one school of thought supports the idea that bleeding comes from Woodruff 's plexus and another group thinks this is mainly from posterior septum. However, it is important to be familiar with what kind of abnormal vasculature could cause bleeding as the patient may not actively bleeding at the time of examination. Furthermore, the bleeding site may not be quite obvious.

\section{Objectives}

1. To study the location of bleeding points in idiopathic epistaxis.

2. To get familiarized with the nasal vascular pattern in Little's area and Woodruff plexus.

3. To study the nasal skeleton in patients with epistaxis.

\section{Methodology}

Adult patients presenting to emergency department or to ENT ward (R side) at Kandy Hospital between February 2014 and august 2014 with epistaxis were included considering the following exclusion criteria. Detailed history, examination and investigations as clinically indicated were performed to rule out secondary causes.

\section{Exclusion criteria}

1. Having a histologically proven Sino nasal malignancies or suspicious symptoms / signs

2. Under 12 years of age

3 Bleeding or coagulation dysfunctions (including medications)

\section{History of nasal surgeries or nasal trauma}

\section{Inclusion criteria}

1. having epistaxis as the main presenting complaints

Patients bleeding actively from a blood vessel visible in anterior rhinoscopy were taken to operating theatre ( through a minor procedure list ) urgently and managed using electrocautery. Patients who were actively bleeding and having negative anterior rhinoscopy were managed by nasal packing using vasline ribbon gauze and packs were kept in situ for 48 hours. Patients who did not bleed actively at the time of presentation were also examined with anterior rhinoscopy and Rigid nasal endoscopy in the operating theatre during the next available list. Endoscopy was done using 0 degree $2.7 \mathrm{~mm}$ (30 degree as well if indicated ) rigid nasal endoscopy. Nasal mucosa was anaesthetized using $10 \%$ lignocaine spray. Small ribbon gauze soaked in $1 ; 100,000$ adrenaline was used if needed. Patients who had a bleeding point were treated with electrocautery. Patients who did not have any bleeding point 
but continue to bleed or who bled after cautery were examined under general anaesthesia and managed with appropriate surgical procedure. All Endoscopic examination was done by the post graduate trainee. Consultant's opinion was taken appropriately. Initial assessment during the admission (general condition and anterior rhinoscopy ) was done by the attending medical officer. Rhinoscopic findings were documented in the data sheet. Ethical clearance was obtained from the Ethical committee, Kandy Hospital.

\section{Results}

27 patients aged between 14 and 85 years were included in this study. Mean age is 49.4 years and median age is 54 years. Bleeding points were identified in 23 patients $(85 \%)$.

\begin{tabular}{|l|c|}
\hline Bleeding from septum- $19 *(82 \%)$ \\
\hline Little's area & 13 \\
\hline Vessel on spur & 1 \\
\hline Posterior septum & 4 \\
\hline Bleeding point in a crevice & 3 \\
\hline
\end{tabular}

\begin{tabular}{|l|l|}
\hline Bleeding from lateral wall $-4(18 \%)$ \\
\hline Anterior to axilla of middle turbinate & 2 \\
\hline Anterior end of MT & 1 \\
\hline Uncinate process & 1 \\
\hline
\end{tabular}

*Two patients had multiple potential bleeding points (Little's area + vessel on a spur, Little's area + vessel on uncinate).

One patient in this group continued to bleed after cautery and he underwent external carotid artery ligation on the following morning.

\begin{tabular}{|l|l|}
\hline No bleeding point - 4 & 2 \\
\hline Spontaneously settled & 2 \\
\hline External carotid artery ligation & 1 \\
\hline septoplasty &
\end{tabular}

Out of 27 patients 2 had symptomatic nasal septal deviation and 6 had prominent septal spur detected during examination.

We noted a plexus like vasculature at the posterior part of the inferior turbinate (Woodruffs plexus) but this was not bleeding and did not look like the source .

A

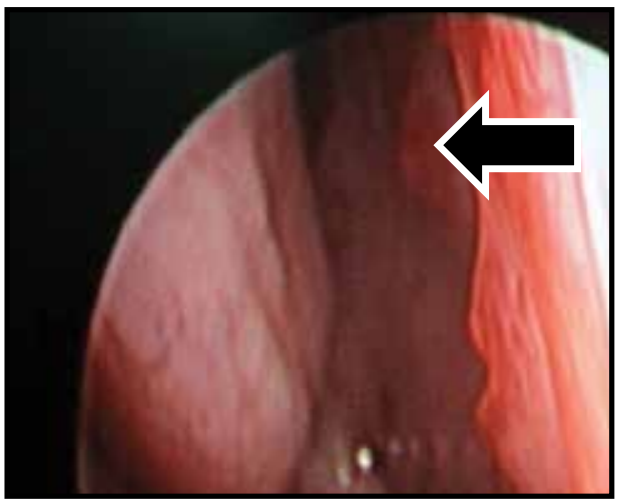

B

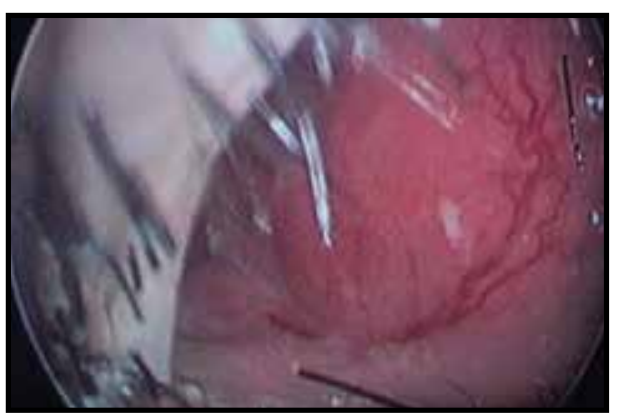

C

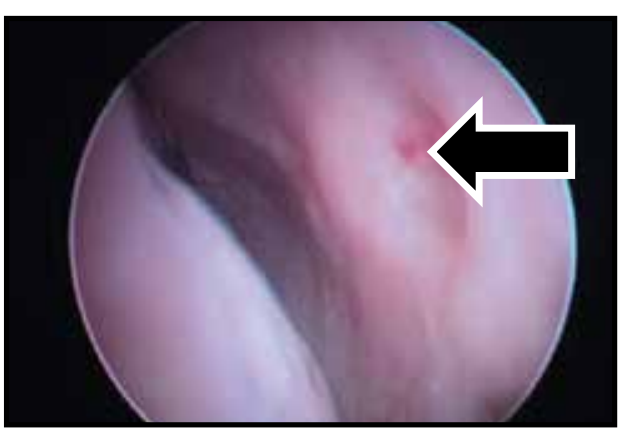

D

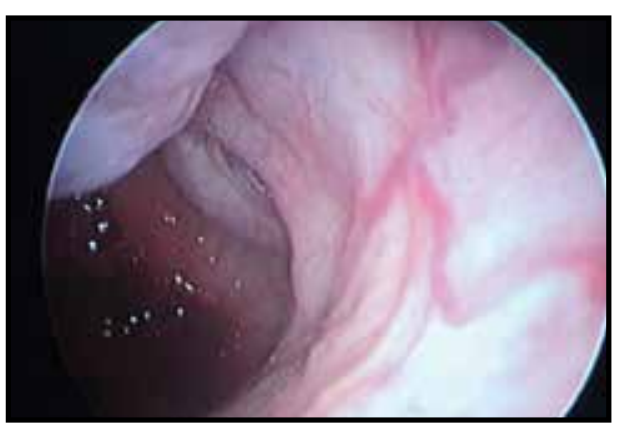

Fig A - Haemorrhagic nodule like vascular abnormality

Fig B - Prominent vessel in Littles's area

Fig C - Bleeding point hidden in a crevice

Fig D - Woodruff's plexus ( left ) 


\section{Discussion}

The most efficient way of treating idiopathic epistaxis is to find out the bleeding point and manage it. Thorough examination of the nasal cavity is the way forward for this. We were able to locate bleeding point in $85 \%$. A large prospective study has noted a detection rate of 94 $\%{ }^{(4)}$ where as a retrospective data collection by Thornton M.A., Mahesh B.N., Lang J has found the bleeding point in $83 \%{ }^{(3)}$. They have only included posterior bleeding. Comparatively, our study sample is bit more heterogeneous. On the other hand, they ${ }^{(4)}$ have done complete nasal endoscopy before packing in every patient. As some of our patients had packing before endoscopy bleeding point might have been concealed by the final examination.

The majority of patients have bled from the septum $(82 \%)$ and in fact, this is comparatively a high value ${ }^{(4)}$. This might be because we have included patients having anterior bleeding as well and commonest reason for anterior bleeding is the septum.

We have also noted that small crevice in septum can contain the bleeding vessel which does not look like a common finding. One report involved 107 patients has declared that they could not see any hidden bleeding sources within such deflections or spurs in the septum ${ }^{(1)}$. But, somewhat similar to this finding has been documented is turbinates ${ }^{(3)}$.

A tiny nodular looking bleeding points which do not look like any prominent vessel as such (Fig. A) been reported as a common abnormality which can be missed easily ${ }^{(1)}$ Very gentle probing over these area can cause brisk bleeding this confirms it as the source of bleeding.

One paper has shown that there is no association between nasal bleeding and septal abnormalities as the bleeding points they had noted was not close to the septal deflection or the spur ${ }^{(1)}$. But in our study one needed septoplasty.

The main draw backs in our study was not incorporating endoscopy before nasal packing , not recruiting a control group to study, the association between nasal skeleton anomalies and epistaxis. Furthermore, as our sample is fairly heterogeneous sometimes it is difficult to compare the finding with samples which are more defined such as studies on posterior epistaxis. Finally, we noted that a careful follow up of these patients in the same hospital with in first $2-3$ weeks is useful to get a better impression about the outcome of the treatment.

\section{Conclusion}

This prospective study has shown the commonest bleeding point in adult epistaxis is located in the septum and this is identifiable and manageable locally without needing invasive surgical treatments. Initial endoscopic examination prior to nasal packing should be encouraged. But a number of patients can also have bleeding points in lateral nasal wall, not necessarily related to Woodruff's plexus. Source of bleeding can be hidden under a septal spur or within a mucosal deflection.

\section{References}

1. Padgham N. Epistaxis: anatomical and clinical correlates. J Laryngology and otology 1990; 104: 308-311.

2. Mcgarry GW.Nasal endoscope in posterior epistaxis: a preliminary evaluation. J Laryngology and otology 1991;105:428-431.

3. Thornton MA, Mahesh BN, Lang J. Posterior epistaxis: identification of common bleeding sites. Laryngoscope 2005; 115: 588-590.

4. Chiu TW, McGarry GW. Prospective clinical study of bleeding sites in idiopathic adult posterior epistaxis. Otolaryngology- Head and Neck Surgery2007; 137: 390-393.

5. Chiu TW, Shaw-Dunn J, McGarry GW. Woodruff's nasopharyngeal plexus : how important is it in posterior epistaxis?. Clinical otolaryngology1998; 23: 272-279.

6. Small M, Maran AGD, Epistaxis and arterial ligation. J Laryngology Otology 1984;98:281-4. 